\title{
Treatment of Alloxan-Induced Diabetic Rats with Metformin or Glitazones is Associated with Amelioration of Hyperglycaemia and Neuroprotection
}

\author{
Oluwole Akinola ${ }^{*}$, Michael Gabriel, Abdul-Azeez Suleiman and Felix Olorunsogbon \\ Department of Anatomy, Faculty of Basic Medical Sciences, College of Health Sciences, University of Ilorin, P.M.B. \\ 1515, Ilorin, Nigeria
}

\begin{abstract}
Neurobehavioural and cognitive impairments are reportedly associated with both types of diabetes mellitus; and the structural and molecular aberrations in support of these are emerging. In the present study, we report the effects of induced diabetes and its treatment with or without oral hypoglycaemic drugs on the morphology and oxidative stress status of the prefrontal cortex. Hyperglycaemia was induced in fasted Wistar rats with alloxan (150 $\mathrm{mg} / \mathrm{kg}$ body weight). Hyperglycaemic rats were treated with or without oral hypoglycaemic drugs (metformin, $150 \mathrm{mg} / \mathrm{kg} / \mathrm{d}$; pioglitazone, 3 $\mathrm{mg} / \mathrm{kg} / \mathrm{d}$; and rosiglitazone, $10 \mathrm{mg} / \mathrm{kg} / \mathrm{d}$ ). At 28 days of treatment, prefrontal morphology was studied by the cresyl fast violet (CFV) and luxol fast blue (LFB) techniques; and malondialdehyde (MDA) and superoxide dismutase (SOD) were assayed in prefrontal homogenate. Blood glucose was estimated by the glucose oxidase method. Prefrontal cortex neurons showed weak affinity for CFV and LFB in the untreated diabetic rats; as opposed to the relatively strong affinity for these stains in the non-diabetic control rats and diabetic rats on oral hypoglycaemic interventions. In the latter, blood glucose was not significantly different $(\mathrm{P}>0.05)$ from the control at 28 days of treatment. Moreover, prefrontal MDA and SOD were not significantly different between all the groups $(\mathrm{P}>0.05)$. These findings suggest that morphologic aberrations are provoked by untreated diabetes mellitus, even in the absence of oxidative stress; and that oral hypoglycaemic interventions are neuroprotective in alloxan-induced diabetic rats.
\end{abstract}

Keywords: Metformin, pioglitazone, rosiglitazone, neuron, neuroprotection, diabetes.

\section{INTRODUCTION}

One of the several complications of diabetes mellitus is neuropathy, which could involve both the central and peripheral nerve tissues. Cerebral involvement in diabetic lesions may manifest as impairment of learning, cognition and memory in human and animals [1]. Diabetes is also a risk factor for vascular dementia and Alzheimer's disease [2].

Recent studies are unravelling the structural and molecular changes characteristic of the brain in chronic diabetes patients and experimental animals. Such changes include smaller volume of the grey matter and relatively high white matter lesions [3]; lower brain-to-intracranial volume ratios [4]; and grey matter atrophy [5] in human subjects. In animal studies of diabetic neuropathy, significant reduction in the density of the dendritic spines of prefrontal pyramidal neurons [6]; neuronal DNA and protein loss in association with brain atrophy [7]; and prefrontal Nissl body deficits [8], have been reported.

However, it has been shown that with optimal glycaemic control, diabetic brain lesions could be prevented and/or ameliorated. In a recent study by Serbedzija et al., [7], treatment of streptozotocin-induced (STZ-induced) diabetic

Address correspondence to this author at the Department of Anatomy, Faculty of Basic Medical Sciences, College of Health Sciences, University of Ilorin, P.M.B. 1515, Ilorin, Nigeria; Tel: 2347032268248;

E-mail:woleakinola@yahoo.com rats with a combination of insulin and insulin-like growth factor I (IGF-I) protected brain tissues from diabetic lesions. Previously, we have also shown that treatment of STZinduced diabetic rats with a botanical intervention from neem and bitter leaf was protective against diabetes brain injury [8].

In the present work, we studied the effect of induced diabetes on the morphology of the prefrontal neurons; as well as on some tissue markers of oxidative stress (malondialdehyde and superoxide dismutase). We also tested the hypothesis that treatment of rats with hypoglycaemic drugs is protective against diabetes-induced brain lesions.

\section{MATERIALS AND METHODS}

\section{Animals}

Male Wistar rats (average weight: $140 \mathrm{~g}$ ) were bred and maintained on rodent chow from Bendel Feed (Ewu, Nigeria). All animals were exposed to an environment of 12 hour light:12 hour dark period, at a room temperature between $23{ }^{\circ} \mathrm{C}$ and $25^{\circ} \mathrm{C}$.

\section{Induction of Hyperglycaemia}

A cohort of male Wistar rats was fasted overnight for at least 8 hours. Hyperglycaemia was induced in each fasted rat by administering alloxan monohydrate $(150 \mathrm{mg} / \mathrm{Kg}$ body weight; intraperitoneal) in normal saline. The control cohort was administered normal saline intraperitoneally. At 7 days post-induction of hyperglycaemia, blood glucose was assayed by the glucose oxidase method, using a glucometer. 
Only those rats with established hyperglycaemia (blood glucose $>300 \mathrm{mg} / \mathrm{dl}$ ) were included for subsequent treatment.

\section{Treatment of Hyperglycaemic Rats with Oral Hypoglycaemic Drugs}

Three oral hypoglycaemic drugs (metformin, rosiglitazone and pioglitazone) were used in the present study. Each drug was administered orally to a cohort of hyperglycaemic rats $(n=8)$ at 7:00 - 9:00 each day for 28 days. Metformin (Merck, Germany) was administered at 150 $\mathrm{mg} / \mathrm{kg}$ body weight/day [9]; rosiglitazone (GlaxoSmithKline, USA) at $3 \mathrm{mg} / \mathrm{kg}$ body weight/day [10]; and pioglitazone (Sun, India) at $10 \mathrm{mg} / \mathrm{kg}$ body weight/day [11]. Untreated diabetic group received only the vehicle (distilled water).

\section{Estimation of Blood Glucose}

In the control, non-treated diabetic, and oral hypoglycaemic-treated rats, blood glucose was assayed weekly by the glucose oxidase method.

\section{Termination of Treatment}

At 28 days of treatment with oral hypoglycaemic drugs, all rats were anaesthetized with ether (Sigma, MO), 24 hours after the last dose of the drugs. Each rat was then decapitated and the brain removed with the brain forceps. For each rat, the prefrontal region (part of the cerebral hemisphere just caudal to the olfactory bulb) was either fixed in formolcalcium or homogenized in phosphate buffered solution $(\mathrm{pH}$ 7.4, 0.1 M).

\section{Bioassays and Histological Processing}

The homogenate from each rat was spun at $500 \mathrm{xg}$ for 10 minutes, in a centrifuge. The supernatant were assayed for total protein and markers of oxidative stress. Protein was assayed by the Biuret method [12]. Tissue malondialdehyde (MDA) and superoxide dismutase (SOD) were assayed by the method of Ohkawa et al., [13] and Misra and Fridovich [14], respectively.
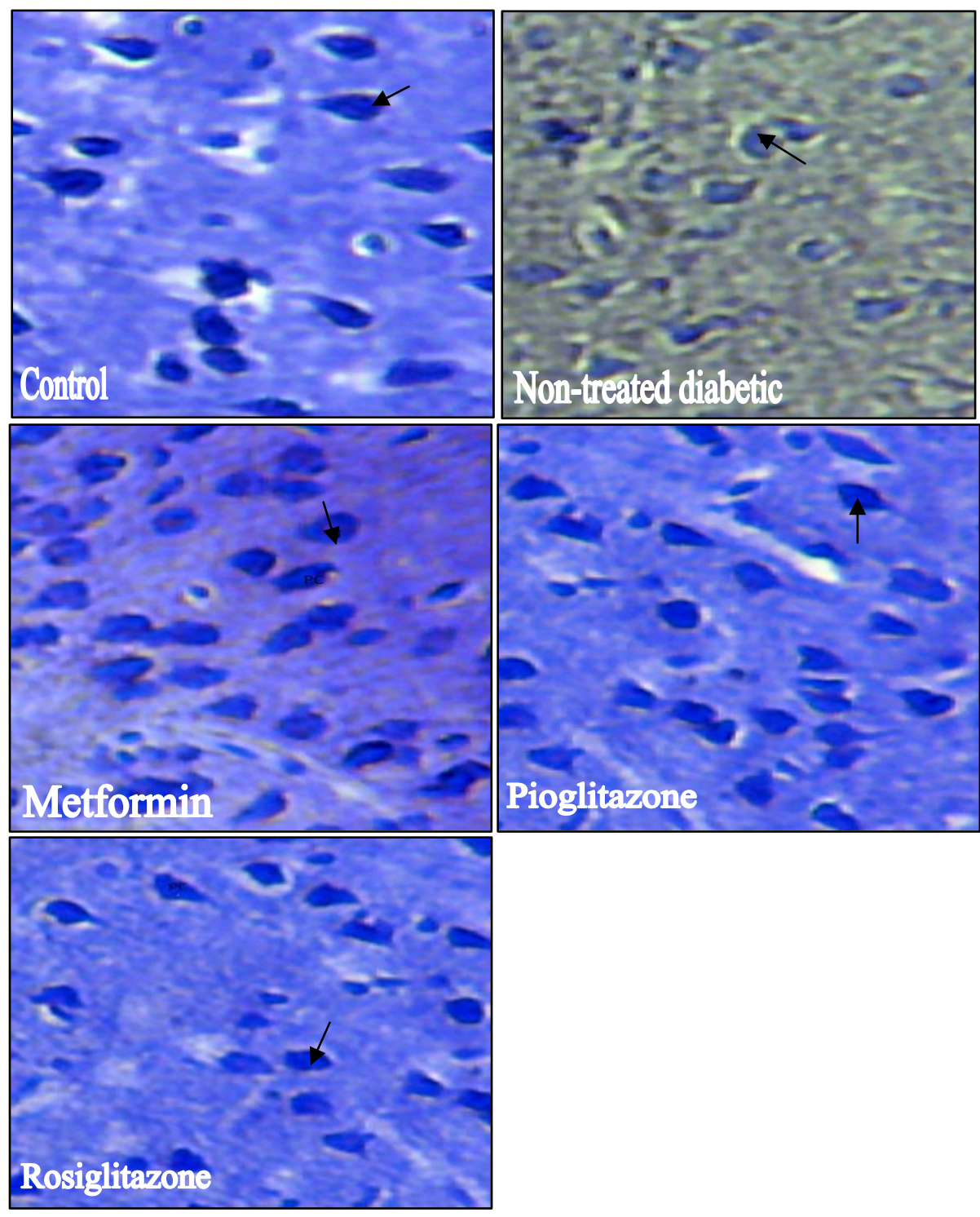

Fig. (1). Photomicrographs of the prefrontal cortex of the control, non-treated diabetic and oral hypoglycaemic-treated rat brain. Chromatolysis is observable in the non-treated diabetic group (thin arrow), in contrast to the well-stained Nissl substance and nuclei of the neurons in the control and oral hypoglycaemic-treated groups. Cresyl fast violet (Magnification, x400). 
Moreover, formol-calcium-fixed prefrontal lobes were dehydrated and embedded in paraffin wax. Eight micrometer-thick sections were cut on a rotary microtome; and sections were either stained by the cresyl fast violet (CFV) or luxol fast blue (LFB) technique, as described by Bancroft and Stephens [15]. Images were captured with an MW1-HD2 digital microscope.

\section{Statistical Analysis}

Data collected on blood glucose and markers of oxidative stress were analysed and presented ad mean \pm standard error of the mean (mean \pm SEM). Means were compared by the analysis of variance, followed by the Bonferroni post-hoc test. $\mathrm{P}<0.05$ was accepted as significant.

\section{RESULTS}

\section{Histologic Findings}

In the non-treated diabetic rats, prefrontal neurons showed poorly-stained nuclei and chromatolysis (poorly- stained Nissl bodies), as demonstrated by the CFV technique (Fig. 1). However, in the non-diabetic control and hypoglycaemic drug-treated diabetic rats, prefrontal cortex neurons showed well-stained nuclei and Nissl bodies.

Similarly, LFB technique showed poorly myelinated axons in the prefrontal cortex of non-treated diabetic rats (Fig. 2). Axonal integrity was however preserved in the nondiabetic control and hypoglycaemic drug-treated diabetic rats (Fig. 2).

\section{Blood Glucose Levels in the Control and Treatment Groups}

The blood glucose responses to oral hypoglycaemic drugs in alloxan-induced diabetic rats are shown in Table $\mathbf{1}$. At 28 day of treatment, no statistically significant elevation in blood glucose levels occurred in all the groups compared to the control rats $(\mathrm{P}>0.05)$, except in the non-treated diabetic rats, where significant elevations in blood glucose occurred $(\mathrm{P}<0.05)$.
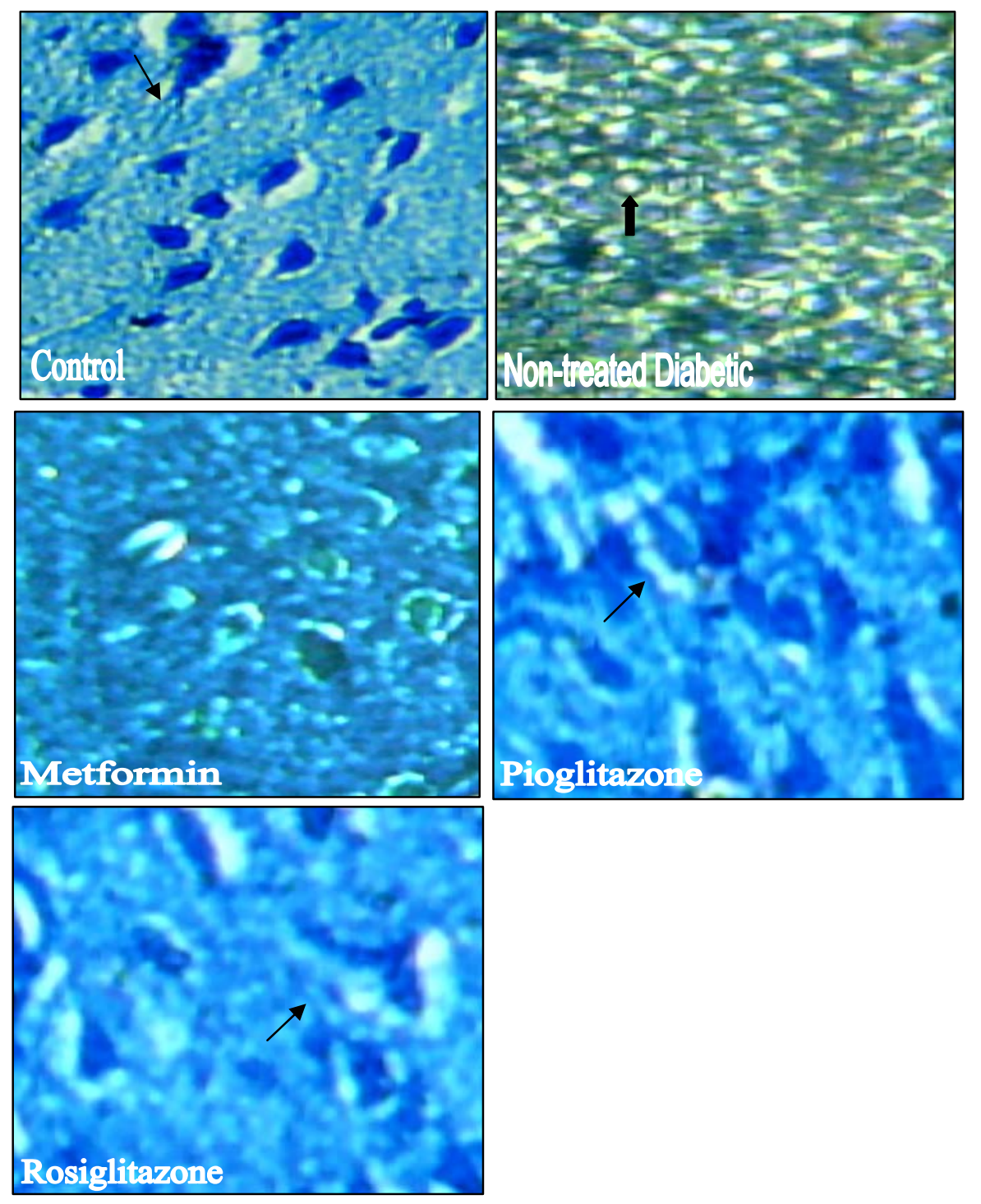

Fig. (2). Photomicrographs of the prefrontal cortex of the control, non-treated diabetic and oral hypoglycaemic-treated rat brain. Loss of myelin sheath is observable in the non-treated diabetic group (thick arrow), in contrast to the intact myelin sheath of the control and hypoglycaemic drug-treated groups (thin arrow). Luxol fast blue (Magnification, x400). 
Table 1. Effect of Oral Hypoglycaemic Drugs on Blood Glucose Levels of Alloxan-Induced Diabetic Rats (mg/dl)

\begin{tabular}{|c|c|c|c|c|c|c|}
\hline & n & Week 0 & Week 1 & Week 2 & Week 3 & Week 4 \\
\hline \hline Control & 8 & $110 \pm 5$ & $105 \pm 3$ & $130 \pm 6$ & $123 \pm 4$ & $125 \pm 2$ \\
\hline Non-treated Diabetic & 8 & $480 \pm 20 \mathrm{a}$ & $450 \pm 23 \mathrm{a}$ & $460 \pm 19 \mathrm{a}$ & $370 \pm 15 \mathrm{a}$ & $410 \pm 26 \mathrm{a}$ \\
\hline diabetic + metformin & 8 & $380 \pm 16 \mathrm{a}$ & $400 \pm 20 \mathrm{a}$ & $160 \pm 8 \mathrm{~b}$ & $155 \pm 9 \mathrm{~b}$ & $150 \pm 10 \mathrm{~b}$ \\
\hline Diabetic + pioglitazone & 8 & $400 \pm 16 \mathrm{a}$ & $390 \pm 18 \mathrm{a}$ & $400 \pm 20 \mathrm{a}$ & $140 \pm 9 \mathrm{~b}$ & $180 \pm 4$ \\
\hline Diabetic + rosiglitazone & 8 & $410 \pm 17 \mathrm{a}$ & $480 \pm 19 \mathrm{a}$ & $140 \pm 11 \mathrm{~b}$ & $95 \pm 9 \mathrm{~b}$ & $135 \pm 6 \mathrm{~b}$ \\
\hline
\end{tabular}

Data are mean \pm SEM (mg/dl); $\mathrm{a}=\mathrm{P}<0.05$ compared with control; $\mathrm{b}=\mathrm{P}<0.05$ compared with non-treated diabetic

\section{Prefrontal Malondialdehyde and Superoxide Dismutase}

Prefrontal MDA levels and SOD activities were not significantly different $(\mathrm{P}>0.05)$ between the non-diabetic control and the treatment groups (Table 2). Similarly, MDA levels were not significantly different between the nontreated diabetic rats and the hypoglycaemic drug-treated groups $(\mathrm{P}>0.05)$. Moreover, SOD activity in all the treatment groups were not significantly different from the non-diabetic control rats $(\mathrm{P}>0.05)$, as shown in Table 2 . However, SOD activity in the pioglitazone-treated diabetic group was significantly higher than the value in the non-treated diabetic rats $(\mathrm{P}<0.01)$ (Table 2$)$.

\section{DISCUSSION}

The chronological progression of diabetes mellitus is associated with varying degrees of cognitive deficits in human [16] and animals [17]. The structural changes and mechanisms underlying such diabetes-related neurocognitive impairment are also evolving.

Hyperglycaemia and hypoglycaemia are both risk factors for neurostructural and cognitive impairment of diabetes mellitus. Previous morphologic studies of the brain in diabetic human and animals showed structural impairment of both the white and grey matter. In the recent study of Novak et al., [5] in human subjects, the diabetic brain showed atrophic changes in the grey matter of the frontal, temporal and parietal lobes. This agrees with the findings of Manschot et al., [18], which showed brain atrophy in diabetic subjects using magnetic resonance imaging (MRI) technique.

In the present work, we studied brain (prefrontal cortex) morphology in alloxan-induced diabetic rats with and without treatment with oral hypoglycaemic drugs. Histologic study of the brain of the non-treated diabetic rats showed structural impairment characterised by loss of axonal myelin sheath and poor Nissl staining outcome. The latter suggests loss of Nissl substance and nuclear DNA in the somata of the diabetic brain. Thus, the present morphologic findings from animal studies are empirical evidence of the structural changes of the brain in diabetic conditions.

Although neurobehavioural and cognitive tests were not performed in the present animal study, the observed morphologic impairment could provoke cognitive and other functional deficits of the brain in the non-treated diabetic rats. In a related study by Serbedzija et al., [7], DNA loss in brain neurons was reported in streptozotocin-induced diabetic rats. Our finding of poorly-stained nuclei of prefrontal neurons, as shown by the CFV technique (Fig. 1) thus corroborates the report of Serbedzija et al., [7]. It also agrees with the previous findings in our laboratory [8].

In addition, the involvement of myelinated axons in the morphologic impairment induced by an untreated diabetic state is evident in the present study. Loss of myelin sheath of axons was demonstrated by the LFB technique (Fig. 2). Previous study using the Golgi technique showed significant reduction in the mean density of pyramidal neuron dendritic spines of the medial prefrontal cortex after two months of untreated diabetes. The latter finding, and our observations in the present study, support the report of diabetes-induced brain lesion by previous workers $[3,5,18]$.

Furthermore, in the present study, the treatment of alloxan-induced hyperglycaemic rats with oral hypoglycaemic drugs (metformin, pioglitazone and rosiglitazone) protected the animals from the prefrontal lesions observed in the non-treated diabetic rats (Figs. 1, 2). This neuroprotective effect of oral hypoglycaemic interventions was possibly the result of optimum glycaemic control. Glycaemic study showed no significant difference in the blood glucose levels of the non-diabetic control and oral hypoglycaemic-treated rats at 28 days (Table 1). Moreover, blood glucose levels of the non-treated diabetic rats were consistently higher than the hypoglycaemic drug-treated

Table 2. Effect of Oral Hypoglycaemic Drugs on the Prefrontal SOD and MDA of Alloxan-Induced Diabetic Rats

\begin{tabular}{|c|c|c|c|}
\hline & $\mathbf{n}$ & SOD (U/mg Protein) & MDA (nmol/mg Protein) \\
\hline \hline Control & 8 & $365.4 \pm 24.0$ & $1.11 \pm 0.22$ \\
\hline Non-treated diabetic & 8 & $389.0 \pm 43.1$ & $0.84 \pm 0.15$ \\
\hline Diabetic + metformin & 8 & $332.9 \pm 49.3$ & $0.69 \pm 0.10$ \\
\hline Diabetic + pioglitazone & 8 & $459.2 \pm 43.8 *$ & $0.84 \pm 0.16$ \\
\hline Diabetic + rosiglitazone & 8 & $376.6 \pm 6.8$ & $0.73 \pm 0.11$ \\
\hline
\end{tabular}


treated groups. This suggests that optimum glycaemic control protects against neural lesions in diabetic rats. A recent study by Serbedzija et al., [7] also showed neuroprotection by insulin and insulin-like growth factor I (IGF-I) against brain atrophy in streptozotocin-induced diabetic rats.

Moreover, in human studies, optimum glycaemic control improved cognitive and social functions in diabetic subjects [19]. These latter findings in diabetic subjects, and our histologic observations in the present animal study, underscore the importance of optimum glycaemic control to brain morphology and functions in the diabetic state.

Furthermore, results from the present oxidative stress studies in diabetic rats suggest that the structural lesions observed in the non-treated diabetic rats were not the consequence of oxidative damage. MDA and SOD levels were not significantly different from the control in all the treatment groups (Table 2). Thus, it is unlikely from the present data that oxidative stress contributed to the neural lesions seen in the non-treated diabetic rats. That is, the data from the present animal study suggests that neural lesions could occur in the diabetic state in the absence of oxidative stress.

In conclusion, data from the present study shows that neural lesions are induced by untreated diabetic mellitus, even in the absence of oxidative stress; and that metformin and glitazones are neuroprotective in alloxan-induced diabetic rats.

\section{ACKNOWLEDGEMENTS}

None declared.

\section{CONFLICT OF INTEREST}

Authors declare no conflict of interest.

\section{REFERENCES}

[1] Parihar MS, Chaudhary M, Shetty R, Memnani T. Susceptibility of hippocampus and cerebral cortex to oxidative damage in streptozotocin-treated mice: prevention by extract of Withania somnifera and Aloe vera. J Clin Neurosci 2004; 11: 397-402.

[2] Luchsinger JA, Tang MX, Stern Y, Shea S, Mayeux R. Diabetes mellitus and risk of Alzheimer's disease and dementia with stroke in a multiethnic cohort. Am J Epidemiol 2001; 154: 635-41.
[3] Jongen C, van der Grond J, Kappelle LJ, et al. Automated measurement of brain and white matter lesion volume in type 2 diabetes mellitus. Diabetologia 2009; 50: 1509-16.

[4] Christman AL, Vannorsdall TD, Pearlson GD, Hill-Buggs F, Schretlen DJ. Cranial volume, mild cognitive deficits and functional limitations associated with diabetes in a community simple. Arch Clin Neuropsychol 2010; 25: 49-59.

[5] Novak V, Zhao P, Manor B, et al. Adhesion molecules, altered vasoreactivity and brain atrophy in type 2 diabetes. Diabetes Care 2011; 34: 2438-41

[6] Joghataie M, Roghani M, Jalali M, Baluchnejadmojarad T, Sharayeli M. Dendritic spine changes in medial prefrontal of male diabetic rats using Golgi impregnation method. Arch Iranian Med 2007; 10 (1): 54-8.

[7] Serbedzija P, Madl JE, Ishii DN. Insulin and IGF-I prevent brain atrophy and DNA loss in diabetes. Brain Res 2009; 1303: 179-94.

[8] Akinola OB, Omotoso GO, Dosumu OO, Akinola OS, Olotufore F. Diabetes-Induced Prefrontal Nissl Substance Deficit and the effects of Neem-Bitter Leaf Extract Treatment. Int J Morphol 2011; 29 (3): 850-6.

[9] Majithiya JB, Balaraman R. Metformin reduces blood pressure and restores endothelial functions in aorta os streptozotocin-induced diabetic rats. Life Sci 2006; 78 (22): 2615-24.

[10] Yue TL, Bao W, Gu JL, et al. Rosiglitazone treatment in Zucker diabetic fatty rats is associated with ameliorated cardiac insulin resistance and protection from ischaemia/reperfusion-induced myocardial injury. Diabetes 2005; 55: 554-62.

[11] Ko GJ, Kang YS, Han SY, et al. Pioglitazone attenuates diabetic nephropathy through an anti-inflammatory mechanism in type 2 diabetic rats. Nephrol Dial Transplant 2008; 23 (9): 2750-60.

[12] Weichselbaum WE. An accurate and rapid method for the determination of protein in small amounts of blood serum and plasma. Am J Clin Path 1946; 10: 40-9.

[13] Ohkawa H, Ohishi N, Yagi K. Assay for lipid peroxides in animal tissues by thiobarbituric acid reaction. Anal Biochem 1979; 95 (2): 351-8.

[14] Misra HP, Fridovich I. The role of superoxide anion in the autoxidation of epinephrine and a simple assay for superoxide dismutase. J Biol Chem 1972; 247 (10): 3170-5.

[15] Bancroft J, Stevens A. Theory and practice of histological techniques. . $2^{\text {nd }}$ ed. New York: Churchill Livingston 1982; pp. $131-5$.

[16] Arvanitakis Z, Benett DA, Wilson RS, Barnes LL. Diabetes and cognitive system in older black and white persons. Alzheimer Dis Assoc Disord 2010; 24 (1): 37-42.

[17] Kuhad A, Selthi R, Chopra K. Lycopene attenuates diabetesassociated cognitive decline in rats. Life Sci 2008; 83 (3-4): 12834.

[18] Manschot SM, Brands AMA, van der Grond J, et al. Brain magnetic resonance imaging correlates of impaired cognition in patients with type 2 diabetes. Diabetes 2006; 55 (4): 1106-13.

[19] Okura T, Heisler M, Langa KM. The association of cognitive function and social support with glycaemic control in adulta with diabetes. J Am Geriatr Soc 2009; 57 (10): 1816-24.

This is an open access article licensed under the terms of the Creative Commons Attribution Non-Commercial License (http://creativecommons.org/licenses/ by-nc/3.0/) which permits unrestricted, non-commercial use, distribution and reproduction in any medium, provided the work is properly cited. 